\title{
Influence of the glass transition on the liquid-gas spinodal decomposition
}

\author{
Vincent Testard, Ludovic Berthier, and Walter Kob \\ Laboratoire Charles Coulomb, UMR 5221 CNRS and Université Montpellier 2, Montpellier, France
}

(Dated: November 11, 2018)

\begin{abstract}
We use large-scale molecular dynamics simulations to study the kinetics of the liquid-gas phase separation if the temperature is lowered across the glass transition of the dense phase. We observe a gradual change from phase separated systems at high temperatures to nonequilibrium, gel-like structures that evolve very slowly at low temperatures. The microscopic mechanisms responsible for the coarsening strongly depend on temperature, and change from diffusive motion at high temperature to a strongly intermittent, heterogeneous and thermally activated dynamics at low temperature, leading to logarithmically slow growth of the typical domain size.
\end{abstract}

PACS numbers: 05.70.Ln, 64.70.Pf, 64.75.Gh

The kinetics of phase separation is a fascinating subject with a long history in condensed matter physics [1]. Although this nonequilibrium phenomenon is complex, universal scaling behaviour have been indentified and can be studied using statistical mechanics [2]. Phase separation kinetics also has important experimental and technological consequences [3]. While this phenomenon is well-understood for simple materials, more complex behaviour can be expected if the different phases have specific properties, such as viscolelastic polymer solutions or colloidal suspensions, which interfere and possibly modify the coarsening process in a non-trivial manner.

Here we study numerically the kinetics of the liquid-gas spinodal decomposition over a broad temperature range encompassing the glass transition of the dense fluid. Not much is known about the resulting gas-glass spinodal decomposition-a specific instance of a viscoelastic phase separation [3]-, although this interplay is invoked in a number of contexts [3, 4. Further interesting cases are colloids [5] and proteins [ [6] with short-ranged attraction that form a gel, instead of phase separating. A plausible interpretation is that the dense phase is actually a glass, and this 'arrests' the phase separation [5, 6]. It has recently been suggested that similar structures could be obtained in $\mathrm{C}_{60}$ molecules 7], and that the range of the potential is important [8]. Bicontinous disordered structures reminiscent of the ones obtained in spinodal decompositions may also be found in colourful bird feathers, and were recently interpreted as incompletely phase separated polymeric glasses [9]. Although it can be expected that the glass transition slows down the spinodal decomposition, the general mechanisms at play have never been studied in any detail on the microscopic scale.

Although coarsening processes are most efficiently studied theoretically using coarse-grained models, such as model $\mathrm{H}$ for the liquid-gas spinodal decomposition [1], in practice it is difficult to faithfully incorporate the complex - typically highly nonlinear and history dependent - physical properties of glasses in such descriptions [10]. Thus, we work in the opposite direction, and start from a microscopically realistic description of the homogeneous glass and study its behaviour during phase separation. Indeed, numerous successful atomicscale simulations of the liquid-gas spinodal decomposition exist [11 14]. In Ref. 15], a Lennard-Jones system was quenched to low temperature in the coexistence region, and the resulting gas-crystal phase separation was studied, but no arrest was reported. Simulations of realistic colloidal interactions were reported 16 18], but the quenches have been performed at very low temperatures where particle aggregation are nearly irreversible and thermal fluctuations play little role.

We use molecular dynamics simulations to study a 80:20 binary Lennard-Jones mixture with interaction parameters chosen to yield excellent glass-forming ability [19]. Specific attention was paid here to system sizes. While numerical studies of the glass transition in the homogeneous liquid typically require simulating about $10^{3}$ particles, we found that up to $10^{6}$ particles were needed to obtain results devoid of finite size effects during the phase separation. We obtained most of our quantitative results using $3 \cdot 10^{5}$ particles. We study the kinetics of the phase separation following an instantaneous quench at constant density from the high temperature fluid phase to the coexistence region, which we determined for the present system. In the following we use Lennard-Jones units corresponding to the majority component, expressing length in units of particle diameter, $\sigma$, and time in units of $\tau=\sqrt{m \sigma^{2} / \epsilon}$, where $m$ is the particle mass, and $\epsilon$ the energy scale in the Lennard-Jones interaction.

In Fig. 1 we present a density, $\rho$, and temperature, $T$, phase diagram showing the location of the homogeneous gas, liquid, and glass phases of the system, as well as the coexistence region. For the glass 'transition', we show the location of the temperature $T_{\text {mct }}$ obtained numerically [20] from fitting the temperature evolution of the relaxation time of the system using predictions from the mode-coupling theory. Usually, it is hard to maintain thermal equilibrium below $T_{\mathrm{mct}}$, which can be taken as a proxy for the 'computer' glass temperature. Figure 1 demonstrates that the present model exhibits the right ingredients, as in a quench below $T \approx 0.3$ in the coexis- 


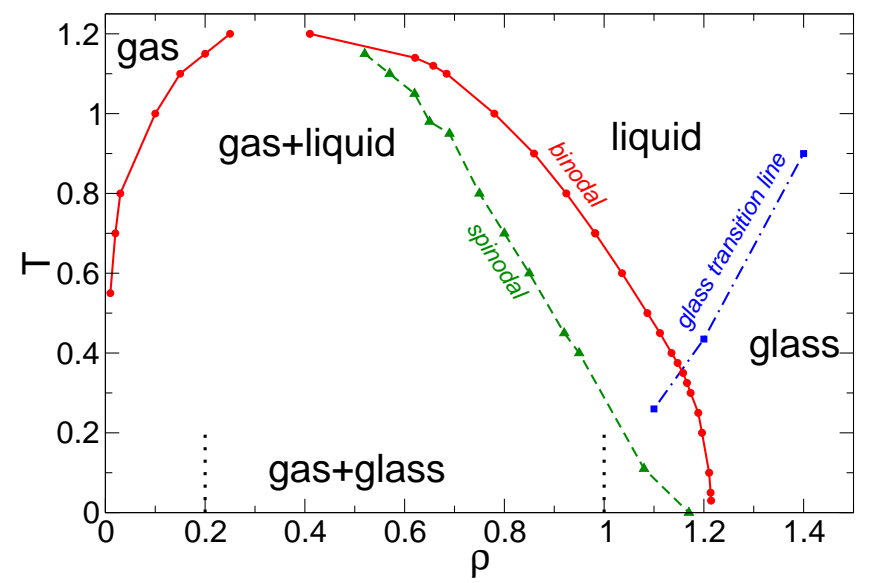

FIG. 1: Phase diagram of the binary Lennard-Jones mixture showing the location of the homogeneous gas, liquid, and glass phases, and the gas-liquid and gas-glass coexistence regions. The glass line corresponds to the mode-coupling temperature (Ref. [20]), while the spinodal is taken from Ref. [21]. The vertical dashed lines separate disconnected droplets from bicontinuous structures.

tence region, the dense phase is a glass - not a liquid.

On the liquid side, the coexistence line hits the glass line near $(\rho \approx 1.15, T \approx 0.3)$. The glass line can be followed between the binodal and the spinodal, where the homogeneous liquid is metastable. Using methods described below, we found that the coexistence line is only weakly affected by the glass line, as found in some experiment [5]. We do not find a reentrant coexistence line slaved to the glass line, as reported in Ref. [ [6]

In Fig. 2 we present typical configurations obtained in various parts of the phase diagram. Figure 2 a shows a homogeneous configuration obtained in the liquid phase, while Fig. 2b shows the final configuration obtained after a quench to $(T=0.5, \rho=0.4)$, where the system is fully demixed at long times. It is in this temperature regime that the liquid-gas spinodal decomposition is typically studied 11 14]. For a quench to densities below $\rho \approx 0.2$ we find that the coarsening proceeds via growth of disconnected droplets, as illustrated in Fig. 2. Similarly, we find that for densities above $\rho \approx 1.0$, the coarsening proceeds via the growth of disconnected gas bubbles immersed in a dense fluid. More interesting is the low temperature regime in the density range $\rho \in[0.2,0.8]$ where bicontinuous, percolating, gel-like structures are obtained at long times, see Fig. 2 $\mathrm{d}$. This means that phase separation is incomplete when $T$ is low, at least in the timescale of the simulation. These configurations are strikingly reminiscent of the confocal microscopy images in colloidal gels [5, 8 ] .

To study quantitatively the phase separation kinetics, we must characterize the structures shown in Fig. 22 d and follow their time dependence for a quench at a given state point. Phase separating systems are usually character-
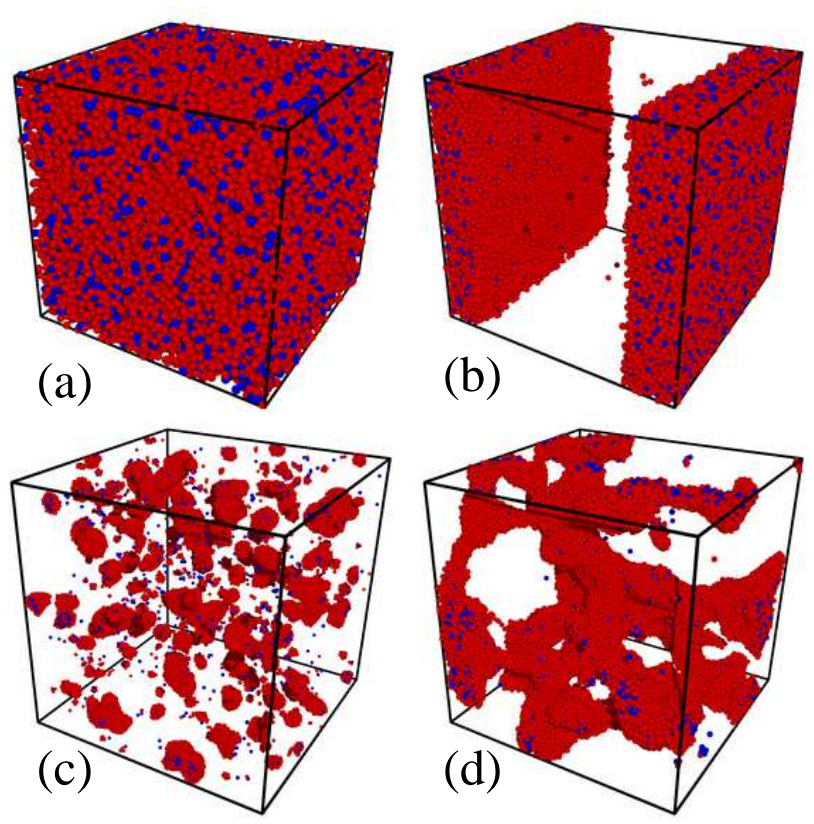

FIG. 2: Representative structures of (a) the liquid phase at $(T=1.75, \rho=0.6)$, (b) a fully demixed system at $(T=0.5, \rho=0.4),(\mathrm{c})$ disconnected growing droplets at $\left(T=0.1, \rho=0.1, t=10^{4}\right)(\mathrm{d})$ a bicontinous gel-like structure obtained at long time at $\left(T=0.1, \rho=0.2, t=10^{4}\right)$ where phase separation is incomplete.

ized in numerical work using the static structure factor of density fluctuations, or equivalently, the pair correlation function. However, we found that a numerically more efficient characterization of the bicontinuous structures is provided by the chord length distribution of the low-density phase, which contains detailed information about the domain size distribution. This is a standard characterization tool for porous media [22, 23].

To measure this quantity, we must first numerically determine the location of the interfaces separating the two phases. This requires a non-trivial coarse-graining for a particle-based simulation in which complex topologies are present. Thus, we discretize space in cubes of small linear size $d=0.5 \sigma$, and define on this cubic lattice a coarse-grained density field, $\bar{\rho}(\mathbf{r})$, using a weighted average over cells around $\mathbf{r}$. The distribution of the coarsegrained density, $p(\bar{\rho})$, is typically bimodal, as expected for a two-phase system. In particular, $p(\bar{\rho})$ exhibits at large $\bar{\rho}$ a maximum, which provides an accurate measure of the average density of the dense phase. We have used this maximum to determine the coexistence line at low temperatures in the phase diagram of Fig. 1 Moreover, a careful analysis of $p(\bar{\rho})$, backed by direct visualizations, shows that a density threshold can be estimated to accurately locate gas and fluid domains, and the position of the interfaces separating them.

The time evolution of the so obtained interfaces is shown in Fig. [3 after a quench at $\rho=0.4$ in the coex- 

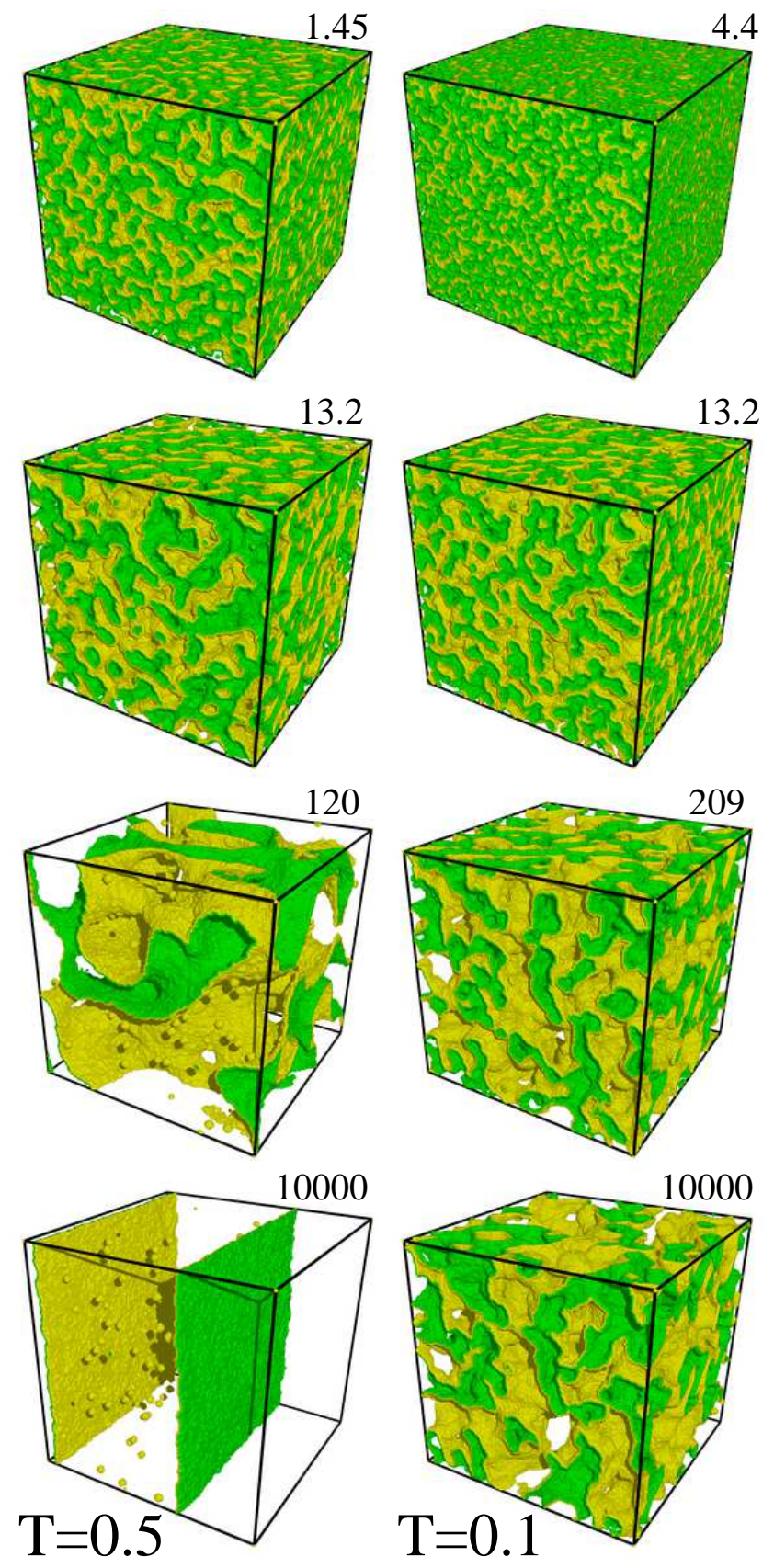

FIG. 3: Time series for a quench at $\rho=0.4$ showing interfaces, the yellow/green side being inside the gas/fluid phase. While the liquid-gas spinodal decomposition at $T=0.5$ proceeds until complete phase separation, dynamics is strongly slowed down in the gas-glass case at $T=0.1$.

istence region. At $T=0.5$, the spinodal decomposition proceeds rapidly until complete phase separation of the system. By contrast, for $T=0.1$ the evolution is similar to the one at $T=0.5$ at short times, $t<10^{2}$, but further evolution is strongly suppressed (but not fully arrested) over the remaining two decades of the simulations, up to $t=10^{4}$.

We are now in a position to measure the chord length

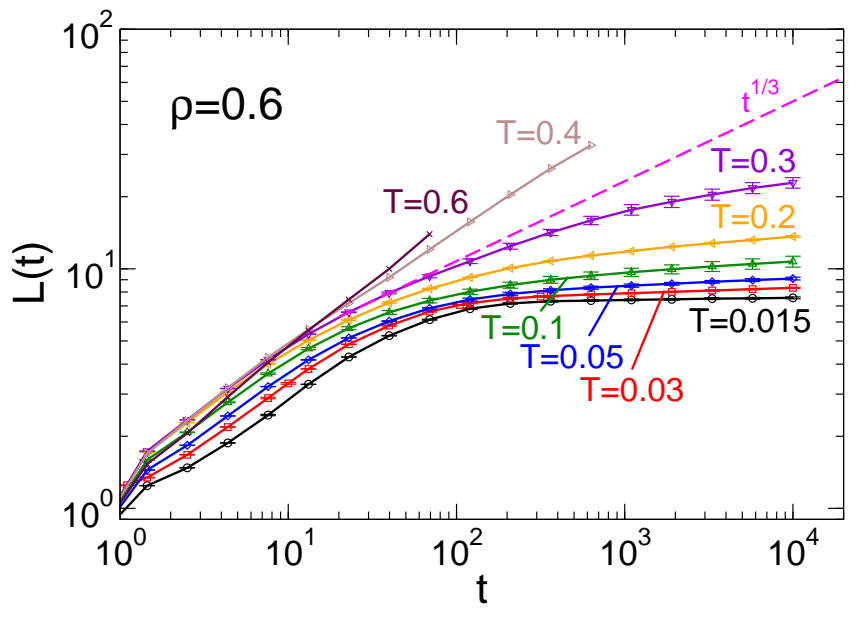

FIG. 4: Evolution of the typical domain size at $\rho=0.6$ and various $T$, in a $\log -\log$ plot. Below $T \approx 0.3$, the growth is much slower than the diffusive law, $L(t) \sim t^{\frac{1}{3}}$ (dashed line), but not fully arrested.

distribution and its evolution. Chords are defined by two consecutives intersections of a straight line with the interfaces of the two-phase material. In practice, we measure chords along the three axis of the lattice used to coarse-grain the density, and measure the length $\ell$ of the segments belonging to the gas phase. Thus, we obtain for each configuration a distribution $P(\ell, t)$. We find that $P(\ell, t)$ has a maximum, and beyond the maximum is well-described by an exponential tail, as commonly found in porous media [22, 23]. We define the average domain size as the first moment of the distribution: $L(t)=\int_{0}^{\infty} \ell P(\ell, t) d \ell$. We find that $L(t)$ is a robust and efficient measure of the typical domain size, which could be easily implemented in confocal microscopy experiments [5], 8].

In Fig. 4 4 we report the time evolution of $L(t)$ at density $\rho=0.6$ and various temperatures from $T=0.6$ down to very low temperatures, $T=0.015$. When temperature is large, we find that the growth law is well described by a power-law, $L(t) \sim t^{n}$, with an apparent exponent $n \approx \frac{1}{2}[11-13]$. We interpret this behaviour as the indication that our data are taken in the crossover between the diffusive $\left(n=\frac{1}{3}\right)$ and hydrodynamic $(n=1)$ regimes, which are notoriously difficult to disentangle in molecular dynamics simulations [14, 24].

The most important observation in Fig. 4 is that for $T<0.3$ the growth law is always much slower than the diffusive $t^{1 / 3}$ law and strongly depends on temperature, as reported in a recent colloidal experiment [8]. The data are actually curved in a log-log representation, suggesting that the growth is not algebraic, but presumably logarithmic. We emphasize that these data are free of finite size effects. Indeed we found that smaller system sizes yield asymptotic lengthscales that are smaller, and spinodal decomposition appears to be much more strongly 

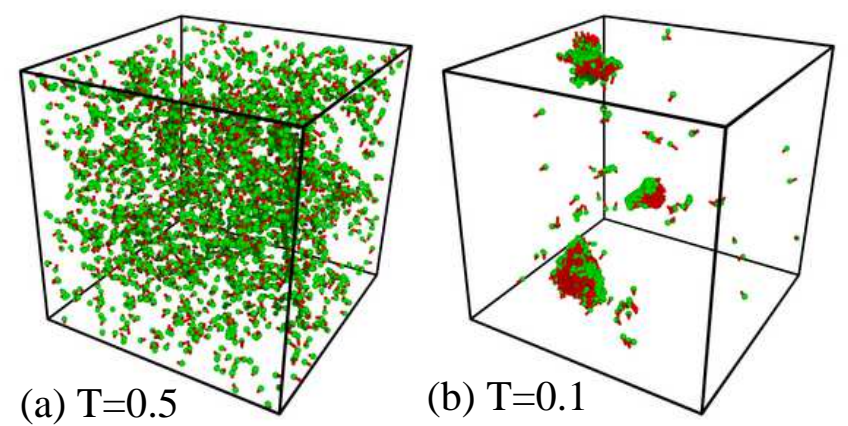

FIG. 5: Mobile particles (green spheres) and their displacements (red cylinders) at $\rho=0.4$ for time intervals corresponding to a equivalent domain sizes. (a) $T=0.5$, times $t=32$ and $t=34.5$. (b) $T=0.1$, times $t=1096$ and $t=1905$.

slowed down at low $T$ if $N$ is chosen too small, a fact which is experimentally relevant for gelation in confinement [25]. A closer look at the data at large times and low $T$ in Fig. 4 shows that if the growth is much slower than diffusive, it is not completely suppressed either, that is, spinodal decomposition is not kinetically arrested by the glass transition of the dense phase, as assumed in previous work [5 7, 16], although it does become difficult to detect at very low temperatures.

Instead, we find that low temperatures, $T<0.3$, strongly modify the microscopic mechanisms of the phase separation. While for the liquid-gas phase separation particles diffuse to relax the curvature of the interfaces, at low $T$ surface tension seems to play very little role. Direct visualization shows that curved interfaces quickly solidify and hardly relax, while particle diffusion in the dense phase (an aging glass) is strongly suppressed.

Particle displacements help to understand the microscopic mechanisms at play. In a given time interval, we select about $1 \%$ of the particles that have moved farthest. At high $T$ we find displacements as in Fig. 5 a where mobile particles appear to be randomly scattered throughout the system, with uncorrelated displacements. The picture is very different at low $T$, see Fig. 5 b, which reveals strong clustering of the fast moving particles, with very correlated displacements. At low temperature, particles in the dense glassy domains are basically arrested and can only move significantly if the whole domain moves as a rigid body. Such large displacements occur in a temporally intermittent and spatially heterogeneous manner, and follow a change in the local topology of the bicontinuous structure, for instance when a thin neck breaks. There are three such relaxation events in the example of Fig. 5 b. We speculate that the main driving force of the phase separation process is the mechanical stress stored in these non-equilibrium, disordered, porous structures, rather than surface tension. Due to thermal fluctuations, these constraints are released in a highly heterogeneous manner, yielding large scale rear- rangements with correlated particle displacements, which ultimately increase the average domain size.

In future work, one should characterize in more detail the microscopic aging dynamics of the system and extend our studies to different kinds of models, as spinodal decomposition represents a promising tool to produce disordered porous media, even in atomic systems.

We thank D. Reichman for initially stimulating this work, B. Coasne, T. Gibaud, and P. Royall for useful discussions. This work is partially funded by ANR Dynhet and Région Languedoc Roussillon. W. K. is a member of Institut Universitaire de France.

[1] P. C. Hohenberg and B. I. Halperin, Rev. Mod. Phys 49, 435 (1977).

[2] A. J. Bray, Adv. Phys. 43, 357 (1994).

[3] H. Tanaka, J. Phys.: Condens. Matter 12, R207 (2000).

[4] S. K. Danchinov, Y. D. Shibanov, and Y. K. Godovsky, Colloid Polym. Sci. 277, 234 (1999).

[5] P. J. Lu, E. Zaccarelli, F. Ciulla, A. B. Schofield, F. Sciortino, and D. A. Weitz, Nature 453, 499 (2008).

[6] F. Cardinaux, T. Gibaud, A. Stradner, and P. Schurtenberger, Phys. Rev. Lett. 99, 118301 (2007).

[7] C. P. Royall and S. R. Williams, preprint submitted to J. Phys. Chem B (2010).

[8] L. J. Teece, M. A. Faers, and P. Bartlett, Soft Matter (in press, 2011).

[9] E. R. Dufresne, H. Noh, V. Saranathan, S. G. J. Mochrie, H. Cao, and R. O. Prum, Soft Matter 5, 1792 (2009).

[10] D. Sappelt and J. Jäckle, Europhys. Lett. 37, 13 (1997).

[11] S. W. Koch, R. C. Desai, and F. F. Abraham, Phys. Rev. A 27, 2152 (1983).

[12] R. Yamamoto and K. Nakanishi, Phys. Rev. B 49, 14958 (1994); Phys. Rev. B 51, 2715 (1995).

[13] E. Velasco and S. Toxvaerd, Phys. Rev. Lett. 71, 388 (1993).

[14] M. Laradji, S. Toxvaerd, and O. G. Mouritsen, Phys. Rev. Lett. 77, 2253 (1996).

[15] B. D. Butler, H. J. M. Hanley, D. Hansen, and D. J. Evans, Phys. Rev. Lett. 74, 4468 (1995).

[16] G. Foffi, C. de Michele, F. Sciortino, and P. Tartaglia, J. Chem. Phys. 122, 224903 (2003).

[17] P. Charbonneau and D. Reichman, Phys. Rev. E 75, 050401(R) (2007).

[18] E. Zaccarelli, J. Phys.: Condens. Matter 19, 323101 (2007).

[19] W. Kob and H. C. Andersen, Phys. Rev. Lett. 73, 1376 (1994).

[20] L. Berthier and G. Tarjus, Phys. Rev. E 82, 031502 (2010).

[21] S. Sastry, Phys. Rev. Lett. 85, 590 (2000).

[22] P. Levitz, Adv. Colloid Interface Sci. 76-77, 71 (1998).

[23] L. D. Gelb and K. E. Gubbins, Langmuir 14, 2097 (1998).

[24] S. Bastea and J. L. Lebowitz, Phys. Rev. Lett. 75, 3776 (1995).

[25] P. S. Sarangapani, Y. Yu, J. Zhao, and Y. Zhu, Phys. Rev. E 77, 061406 (2008).

[26] T. Koyama, T. Araki, and H. Tanaka, Phys. Rev. Lett. 102, 065701 (2000). 